\title{
Acquired Phimosis
}

National Cancer Institute

\section{Source}

National Cancer Institute. Acquired Phimosis. NCI Thesaurus. Code C27149.

Phimosis that develops in an uncircumcised male with a previously loose foreskin. 